\title{
SEADFCON 2017 theme \\ "Quality diabetes care beyond socio cultural barriers"
}

\author{
Maskey R., Gupta PP., Kattel V.
}

Diabetes is emerging as a major global problem worldwide and is reaching epidemic proportions with global prevalence of $8.3 \%$, affecting 387 million adults and costing 612 billion dollars in health care spending in 2014. ${ }^{1}$ The IDF estimates that the prevalence of DMT2 in Nepal was $4.5 \%$ in 2012 and the predicted number of undiagnosed cases in adults was 294 per 1000 population. Relative to neighbouring countries such as Pakistan, Sri Lanka, and Bangladesh, Nepal has a higher prevalence of DMT2 and impaired glucose tolerance. ${ }^{2}$

Diabetes is a particular problem in the South Asian community, as people from this ethnic origin are four times more likely to develop the condition than other groups. ${ }^{3}$ South Asian people with type 2 diabetes also have a greater risk of developing cardiovascular disease and renal problems, and a higher diabetes-related mortality rate is seen among this group than in the general population. ${ }^{4}$

Diabetes especially type 2 diabetes is an emerging major health care problem in Nepal, with rising prevalence and its complications especially in urban populations. Several challenges in diabetes management were identified, including high cost of treatment, limited health care facilities, and lack of disease awareness among patients.

The economic burden of diabetes is enormous. Diabetes is costly because of its chronic nature, the severity of its complications, and the modalities required to control them. ${ }^{5} \mathrm{Consequently,}$ people have frequent and intensive encounters with the health system such as higher use of hospital inpatient care, outpatient visits, emergency visits, and prescription drugs. Moreover, the out-of-pocket expenses associated with diabetes remain a barrier to the prevention of diabetesrelated complications in Nepal. ${ }^{6}$

Culture is a shared and dynamic phenomenon displayed by the behaviours and attitudes of a social group, which remains difficult to interpret, but requires a good understanding by health workers. ${ }^{7}$ It encompasses beliefs, language, social norms and values, including practices which can create a sense of social support and belonging for individuals who share the same core beliefs. These can both facilitate and impede health coping styles, access to and utilisation of healthcare services, and implementation of professional advice. ${ }^{8}$

Prevention or delay of diabetes in this population would improve quality of life and reduce health care costs. Identifying cultural definitions of health and diabetes is critically important to developing effective diabetes prevention programs.

We conclude that a comprehensive national effort is needed to stem the tide of the growing burden of diabetes mellitus type 2 and its complications in Nepal. The government should develop a comprehensive plan to tackle diabetes and other non-communicable diseases supported by appropriate health infrastructure and funding. 


\section{Refrences:}

1. Gyawali B, Ferrario A, Teijlingen E V, Kallestrup P. Challenges in diabetes mellitus type 2 management in Nepal: a literature reviewGlob Health Action. 2016; 10.3402

2. IDF Diabetes Atlas: country estimates table 2011. 2012. Available from: http://www.idf.org/ sites/default/[cited 16 December 2015]

3. Dreyer G, Hull S, Aitken Z, Chesser A, Yaqoob MM. The effect of ethnicity on the prevalence of diabetes and associated chronic kidney disease. QJM. 2009;102:261-9.

4. Osman A, Curzio J. South Asian cultural concepts in diabetes. Nurs Times. 2012;108(28):30-2.

5. Shrestha N, Lohani SP, Angdembe MR, Bhattarai K, Bhattarai J. Cost of diabetes mellitus care among patients attending selected outpatient clinics. JNMA J Nepal Med Assoc. 2013;52:343-8.

6. Upadhyay DK, Palaian S, Shankar PR, Mishra P. Knowledge, attitude and practice about diabetes among diabetes patients in western Nepal. RMJ. 2008;33:8-11.

7. Naeem AG (2003) The role of culture and religion in the management of diabetes: a study of Kashmiri men in Leeds. Journal of the Royal Society for the Promotion of Health 123:11

8. The ethnic group question in the 1991 Census of population. In: Coleman D and Salt J (eds) Ethnicity in the 1991 Census. Volume One. Demographic characteristics of the ethnic minority populations. London: HMSO. 